\title{
Construcción de un índice de satisfacción del turismo en Capulálpam "Pueblo Mágico"
}

\author{
Omar López Ramos* \\ Universidad de la Sierra Juárez (México)
}

\begin{abstract}
Resumen: A fin de evaluar la satisfacción de los turistas en un "Pueblo Mágico", se construyó un índice sumatorio ponderado, mismo que se desagregó en cuatro componentes, obteniéndose las siguientes evaluaciones de satisfacción: Componente de calidad del capital humano (66.7 \%), Componente de calidad de la oferta (20.0\%), Componente de seguimiento de la calidad (66.75 \%) y Componente de percepción de la calidad y satisfacción del turista $(100 \%)$. El promedio ponderado de estos componentes arroja una calificación final para este índice de satisfacción del $63.3 \%$. Los resultados analizados muestran que, en general, la satisfacción de los turistas es ligeramente positiva. Los componentes que son prioritarios de atender tienen que ver con la obtención de certificaciones de la calidad de los productos turísticos ofrecidos, así como con la inserción de capital humano avanzado en turismo, ya que estas dos variables incidieron notablemente en la baja calificación obtenida.
\end{abstract}

Palabras Clave: Pueblo Mágico, Índice satisfacción turismo, Capulálpam

\section{Building a satisfaction index of tourism in Capulálpam "Magic Town".}

Abstract: To evaluate the satisfaction of tourists on a "Magic Town", a weighted summation index was built, same as was disaggregated into four components, it obtained the following assessments of Satisfaction: Component quality of human capital (66.7\%), component quality of supply (20.0\%), component quality monitoring (66.75\%) and component quality perception and satisfaction of tourists (100\%). The analyzed results show that, overall, the satisfaction of tourists is slightly positive. The components that are priorities to attend has to do with obtaining quality certifications of tourism products offered, as well as the inclusion of advanced human capital in tourism, as these two variables significantly influenced the low marks obtained..

Keywords: Magic Town, tourist satisfaction index, Capulálpam.

\section{Introducción.}

Con el objetivo de elevar la competitividad de la oferta turística en México, incrementar el flujo, estadía, así como el gasto promedio del turista, la Secretaría de Turismo (SECTUR) ha buscado la consolidación y fortalecimiento de los destinos turísticos del país mediante diversos programas. Uno de estos es el denominado "Pueblos Mágicos"1 que se empieza a implementar en México a nivel nacional en el año 2001, como una estrategia para el desarrollo turístico orientada a estructurar una oferta turística complementaria y diversificada hacia el interior del país, basada fundamentalmente en los atributos históricos y culturales de localidades singulares (SECTUR, 2014).

Después de trece años del surgimiento de este programa, fue necesario llevar a cabo un diagnóstico para identificar los avances, problemas y oportunidades que se presentan en su operación, a fin de buscar nuevas alternativas para su reestructuración y consolidación como un programa integral, eficaz e independiente, de tal forma que permita establecer un adecuado seguimiento de las acciones y control mediante indicadores que permitan su evaluación con certeza en su impacto turístico.

Profesor-Investigador, Instituto de Estudios Ambientales, Universidad de la Sierra Juárez, Oaxaca, México; E-mail: omarlopezramos@hotmail.com 
El principal trabajo del que se tiene referencia con respecto a la satisfacción de los turistas en un pueblo incorporado al programa de "Pueblos Mágicos" es el estudio realizado durante 2011 y 2012 por la Universidad Anáhuac México Norte (2012), con el apoyo del Fondo Sectorial de Investigación, Desarrollo e Innovación Tecnológica en Turismo dependiente de la SECTUR, este estudio titulado "Evaluación de desempeño de los destinos turísticos en el marco de los Convenios de Coordinación en materia de Reasignación de Recursos", tuvo por objetivo medir el desempeño de tres tipos de destinos turísticos del país (pueblos mágicos, ciudades patrimonio y playas) a través de la construcción de diversos índices, uno de ellos, fue el de satisfacción del turista.

Otro trabajo que analiza la satisfacción de los turistas en un "Pueblo Mágico" fue la "Evaluación del visitante en el Pueblo Mágico de Loreto" realizado por Cruz, Juárez, Cruz y Urciaga (2015), trabajo realizado también a partir de la metodología SECTUR, pero esta emanó de su Centro de Estudios en Turismo $^{2}$. En el presente trabajo, se ha seguido en términos generales el marco teórico del trabajo realizado por la Universidad Anáhuac, especialmente en la referencia a las variables que conforman cada uno de los subíndices construidos, pero a diferencia de ese documento, en el presente, únicamente se ha realizado el análisis para el municipio de Capulálpam de Méndez y no a la totalidad de "Pueblos Mágicos" que enlista SECTUR.

$\mathrm{Al}$ existir una sola unidad de análisis, y al carecer de muchos de los datos necesarios para reconstruir los índices, no fue posible realizar la misma actuación metodológica a las variables que en el documento guía anteriormente mencionado, sin embargo, lo que si se logró, es actualizar muchos de los datos (incluso al año en curso), de tal forma que se muestre la evolución comparativa entre el año en el que el municipio recibe la declaratoria de "Pueblo Mágico" (2008) a una fecha tan actualizada cómo fue posible, a fin de evaluar, mediante la construcción de un índice, cuáles han sido los impactos de esta denominación en la satisfacción del turista.

\section{Contexto del análisis}

Capulálpam de Méndez, es una comunidad indígena de 1,545 habitantes, ubicada en la Sierra Norte de Oaxaca, México. Su oferta turística está enfocada al llamado "turismo de naturaleza" gracias al bosque bien conservado que hay en toda esta región. Además de esto, Capulálpam cuenta con tres monumentos considerados históricos: el Templo de San Mateo Apóstol (del siglo XVI y declarada patrimonio cultural de la humanidad por la UNESCO), el Panteón Municipal (del siglo XIX) y la Plaza Principal (del siglo $\mathrm{XIX),} \mathrm{un} \mathrm{museo} \mathrm{comunitario,} 4$ rutas turísticas diseñadas por la empresa ecoturística comunal, se identificaron 22 actividades de ocio disponibles destacando: la feria del mezcal, feria de pan, concurso de patios y jardines y el aniversario del Pueblo mágico, entre otras.

Respecto a la infraestructura turística, en la comunidad 9 establecimientos ofertan el servicio de hospedaje, con un total de 83 cuartos, lo que representa una capacidad de 283 personas alojadas, 14 establecimientos ofertan el servicio de preparación y venta de alimentos, así también hay transporte disponible (autobuses y taxis colectivos) para arribar al destino.

De acuerdo a datos de la organización comunitaria, Capulálpam registró una afluencia de 13,754 turistas hospedados para el año 2008, lo cual representaba menos del $1 \%$ de los turistas que asistieron al Estado de Oaxaca ese mismo año, y de acuerdo a datos de la Secretaría de Turismo y Desarrollo Económico de Oaxaca (2013), la afluencia de turistas en Capulálpam al finalizar el año 2013 fue de 20,467 turistas, por lo tanto, la tasa de incremento de afluencia turística entre el año 2008 al 2013 fue de $48.8 \%$.

La estadía promedio del turista en la comunidad fue de 1.5 días para el año 2014, y su gasto promedio diario para ese mismo año fue de $\$ 450.00$ por turista UNSIJ (2015).

La evaluación de la satisfacción del turista es pertinente, ya que este proceso de evaluación es un instrumento para mejorar los resultados de cada componente y así mejorar la calidad de gestión de la empresa turística en su conjunto (Chiavenato, 2000), además, permitá detectar necesidades de formación de recursos humanos, optimizar recursos humanos, económicos, elaborar programas de planeamiento y desarrollo congruentes con el crecimiento de la empresa turística (González, 2000). 


\section{Marco teórico del índice de satisfacción.}

Medir la satisfacción representa al menos dos grandes retos: uno a nivel conceptual y otro de tipo metodológico. El primero de estos retos tiene que ver con definir, ¿Qué se entiende por satisfacción? Autores como Hellrigel, Jackson y Slocum (2005: 384), definen la satisfacción como el "estado psicológico que indica cómo se siente una persona respecto a su situación, con base en una evaluación de la situación", mientras que Koontz y Weihrich (2012) definen a la satisfacción como el "gusto que se experimenta una vez que se ha cumplido un deseo".

Un elemento directamente relacionado con la satisfacción de los turistas es la calidad del producto turístico. El éxito de una empresa depende en gran medida de lo que los clientes perciben como diferencias sustanciales (valor añadido) que una empresa realiza respecto a otras que prestan los mismos servicios, esta percepción de valor añadido fideliza clientes (García, 2013: 12).

Por su parte, el factor humano también incide en la calidad del producto entregado al cliente, ya que es el intangible más valioso a la hora de generar valor añadido a los productos y servicios; es también portador de activos intangibles relacionados con el saber hacer (know-how) y es la principal fuente de ventaja competitiva sostenible en las empresas. El personal de una empresa aplica tecnologías, aporta conocimiento, experiencia, creatividad, enriquece, potencia y marca la diferencia corporativa frente a terceros, toma decisiones, estudia el mercado, innova productos o servicios, diseña estrategias y políticas competitivas que permiten la coordinación, la sinergia y la dirección de equipo para que la empresa alcance los objetivos programados y una posición de liderazgo. Empresa, empleados y clientes forman una cadena de valor en las organizaciones de servicios turísticos (García, Fernández y Moreno, 2013: 99).

Calidad, competitividad y satisfacción pues están estrechamente relacionadas, para Dupeyras y MacCallum (2013), la competitividad de un destino turístico es la capacidad para optimizar su atractivo, proveer servicios turísticos de calidad, innovadores y atractivos a los consumidores y ganar cuotas de mercado. La calidad de la experiencia turística no sólo depende de lo que es capaz de ofrecer la empresa privada (hotel, restaurante, etc.), sino del entorno en el que se desarrolla la visita (calidad del entorno urbano, seguridad, condiciones sanitarias, información, etc. (Pedro, 2015).

Por otro lado, en la interacción empresa, empleado y cliente, es este último elemento el que se convierte en el principio y fin del proceso de prestación del servicio. Es el cliente el que percibe en toda su magnitud la calidad de los equipos humanos encargados de prestarle el servicio. Por tanto, es él quien valora y evalúa positivamente esa competencia profesional, decidiendo su fidelidad para con la empresa (García, Fernández y Moreno, 2013: 100).

De la discusión teórica anterior, y en concordancia con los criterios establecidos por la Evaluación del Desempeño de los destinos turísticos (Universidad Anáhuac México Nore, 2012), en la presente evaluación se propone evaluar el índice de satisfacción del turista desagregándolo en cuatro dimensiones o componentes:

1. Componente de calidad del capital humano, medida en base al nivel de educación, cursos y certificaciones con que cuenta la población que directamente atiende al turismo en Capulálpam de Méndez.

2. Componente de calidad de la oferta, medida en base a las certificaciones que tienen los establecimientos que integran la oferta turística de Capulálpam de Méndez.

3. Componente de seguimiento de la calidad.

4. Componente de percepción de la calidad y satisfacción del turista.

Para evaluar estos componentes, se construyó un Índice Sumatorio Ponderado, proceso que se aborda a continuación.

\section{Propuesta metodológica del índice de satisfacción.}

Lazarsfeld (1985: 36-40), postula una estructura a través de la cual pasamos de los conceptos a la elaboración y obtención de índices empíricos. Las cuatro fases mencionadas por este autor son:

1. Representación literaria del concepto, en donde el investigador solo ofrece una construcción, una imagen, abstracta de la realidad;

2. Especificación del concepto, en donde se identifican los componentes, aspectos o dimensiones de los conceptos sociales; 
3. Elaboración de indicadores para la cuantificación de las dimensiones conceptuales descritas;

4. Formación de índices, con los que se sistematiza los datos obtenidos en las etapas precedentes.

Esta estructura fue la guía metodológica utilizada para evaluar los componentes del índice de satisfacción turística de Capulálpam. Debido a la falta de información tanto primaria como secundaria, no se pudo contar con datos que permitieran construir índices más complejos, por esta razón, en el presente análisis, se utilizó uno de los índices más simples que existen, que es el Índice sumatorio ponderado, mismo que mide la agregación de dos o más indicadores parciales, matemáticamente se expresa de la siguiente manera:

$$
\mathrm{aw}_{1}+\mathrm{bw}_{2}+\mathrm{cw}_{3} \ldots+\mathrm{nw}_{\mathrm{n}}=100 \%
$$

Dónde:

$\mathrm{a}=$ Valor de la primera variable.

$\mathrm{b}=$ Valor de la segunda variable.

$\mathrm{n}=$ Valor de la variable enésima

$\mathrm{w}=$ ponderación de la variable enésima.

Como se mencionó en el apartado teórico, el índice de satisfacción del turista, se desagregó en cuatro dimensiones o componentes a los que se les asignó el mismo peso (25.0 \%). Para calcular el valor de cada componente se procedió de la siguiente manera: por ejemplo, el componente de "seguimiento de la calidad" se operacionalizó a través de las siguientes variables:

1. Percepción sobre la calidad en los planes de capacitación turística a través de una revisión general de su contenido.

2. Cuenta con un sistema de información que evalúe la satisfacción de los visitantes.

3. Indicadores de calidad de las evaluaciones de la satisfacción de los visitantes.

La información del valor de estas tres variables se evaluó a través de rúbricas. En el caso de la variable uno, la rúbrica era: positiva, neutral o negativa. Según el tipo de respuesta se le asignaba un porcentaje equivalente al 100, 50 o 0 por ciento, respectivamente. De acuerdo a la información obtenida, la percepción sobre la calidad en los planes de capacitación turística es que estos son malos, por lo que se le asignó el valor de $0.0 \%$.

Para el caso de la variable dos, la opción era de tipo dummy (cumple / no cumple), en caso de cumplir, a esta variable se le asignaba el valor de $100 \%$ en caso contrario, el valor era igual a $0.0 \%$. Dado que para en este caso, el destino turístico sí cuenta con el sistema de información, se le asignó la calificación igual al $100 \%$.

De la misma manera se procedió con la variable tres, en la que se constató que sí cuenta con indicadores de calidad, por lo que se le asignó el valor de $100 \%$. El promedio ponderado para este componente es, entonces, igual a $66.7 \%$ :

$$
\begin{gathered}
\mathrm{aw}_{1}+\mathrm{bw}_{2}+\mathrm{cw}_{3} \ldots+\mathrm{nw}_{\mathrm{n}}=100 \% \\
\mathrm{a} 0.333+\mathrm{b} 0.333+\mathrm{c} 0.333=100 \% \\
(0) 0.333+(100) 0.333+(100) 0.333=66.7 \%
\end{gathered}
$$

Obtenidos de esta manera los cuatro valores para los cuatro componentes, y mediante el uso del índice sumatorio ponderado, se integraron en uno solo índice a fin de obtener la calificación final de la satisfacción del turista, de tal manera que si cumplían satisfactoriamente con los cuatro componentes, la evaluación final fuera igual al 100\%. A los cuatro componentes se les asignó la misma importancia $(25.0 \%)$ y dadas las evaluaciones observadas el valor final de este subíndice fue de $63.3 \%$ :

$$
\begin{gathered}
\mathrm{aw}_{1}+\mathrm{bw}_{2}+\mathrm{cw}_{3} \ldots+\mathrm{nw}_{\mathrm{n}}=100 \% \\
\mathrm{a} 0.25+\mathrm{b} 0.25+\mathrm{c} 0.25+\mathrm{d} 0.25=100 \% \\
(66.7) 0.25+(20) 0.25+(66.7) 0.25+(100) 0.25=63.3 \%
\end{gathered}
$$

A continuación se detalla las variables que integraron cada uno de los componentes, así como los valores obtenidos en fuentes primarias y secundarias. 


\section{Análisis de los componentes del índice de satisfacción.}

5.1. Componente de calidad del capital humano. Para operacionalizar este componente se desintegró en las siguientes variables:

- Grado promedio de escolaridad: mide el número promedio de años de escolaridad de la población. El promedio de escolaridad registrada en Capulálpam de Méndez, para el año 2008 es de 9.6 años, mientras que para el año 2013, se calculó la escolaridad promedio en 10.3 años ${ }^{2}$, lo que significa un crecimiento del $7.1 \%$. La media de escolaridad en la comunidad se encuentra por arriba de la media estatal y nacional, por lo que esta variable se califica como positiva.

- Recursos humanos calificados para atender el sector turismo: mide el total de personas en el destino con formación para laborar en el ramo turístico. Considera recursos humanos calificados a nivel universitario. No se encontraron registros de personas con el grado de Licenciatura en Turismo o área a fin trabajando directamente y de tiempo completo en alguno de los establecimientos que ofrecen servicios turísticos, por lo que esta variable se calificó como negativa.

- Personal del destino con capacitación turística: número de personas que cuentan con capacitaciones en el ramo turístico o que han realizado cursos en temas de prestación de servicios turísticos. De acuerdo a los registros entregados por la regiduría de turismo, se puede constatar que en lo que va del año, se han capacitado 60 personas en distintos temas turísticos, por lo que esta variable se califica como positiva.

- Guías de turistas vigentes certificados: medido en función al número de guías certificados vigentes en el Estado de Oaxaca (general o especializado) por la NOM-08-TUR-2002 y la NOM09-TUR-2002. Se encontró que de un total de cinco guías que prestan estos servicios, solo uno está certificado, debido a lo anterior, esta variable se calificó como negativa.

- Cursos de capacitación turística anual: variable que mide si se han realizado cursos de capacitación turística. De acuerdo a los registros entregados por la regiduría de turismo, en lo que va del año, se han realizado siete cursos de capacitación turística, por lo que esta variable se calificó como positiva.

- Planes de capacitación turística: variable que mide si existen planes de capacitación turística por parte del municipio de Capulálpam. De acuerdo a los registros entregados por la regiduría de turismo, sí existen planes de capacitación turística, por lo que esta variable se calificó positivamente.

De acuerdo a la ponderación de estas seis variables, el valor del componente de calidad del capital humano es igual a $66.7 \%$.

5.2. Componente de calidad de la oferta. Este componente se desintegró en las siguientes variables:

- Participación de establecimientos de alimentos y bebidas en el Programa distintivo H: Mide la existencia de establecimientos de alimentos y bebidas que han acreditado llevar a cabo un manejo higiénico de alimentos, por lo que han obtenido el distintivo H. De acuerdo a la información recabada, solo 2 de los 14 establecimientos que ofrecen alimentos en la localidad cuentan con el distintivo $\mathrm{H}$, por lo que este apartado fue calificado como negativo.

- Empresas con distintivo M: Mide la existencia de las empresas turísticas con el distintivo M, mismo que los acredita como una empresa que ha incorporado a su forma de operar herramientas efectivas y prácticas administrativas que les permitan mejorar la satisfacción de sus clientes, mejorar el desempeño de su personal, mejorar el control del negocio, disminuir los desperdicios, incrementar su rentabilidad y propiciar el desarrollo de una cultura de mejora continua. Ningún establecimiento cuenta con este distintivo, por lo que esta variable se calificó como negativa.

- Cuartos certificados en calidad: Mide la existencia de cuartos de hotel que cuentan con alguna certificación de calidad como: Cristal, ISO9000, ISO9001 y punto limpio, respecto al total del desarrollo turístico. Ningún establecimiento cuenta con este distintivo, por lo que esta variable se calificó como negativa.

- Certificados de calidad de empresas turísticas: Mide la existencia de certificados de calidad en la operación de empresas turísticas. Una sola empresa tiene este certificado, sin embargo, al ser una empresa comunal, concentra el total de la oferta y demanda turística, por lo que esta variable se califica como positiva.

- Certificados otorgados por TripAdvisor: Mide la existencia de establecimientos que han obtenido el galardón que reconoce la excelencia en hostelería, el cual es concedido a los establecimientos 
que han logrado excelentes opiniones de los viajeros de TripAdvisor. Ningún establecimiento cuenta con algún certificado, por lo que esta variable se calificó como negativa.

De acuerdo a la ponderación de estas cinco variables, el valor del componente de calidad de la oferta es igual a $20.0 \%$.

\subsection{Componente de seguimiento de la calidad. Este componente se desintegró en las} siguientes variables:

- Percepción sobre la calidad en los planes de capacitación turística a través de una revisión general de su contenido: Describe las características de los planes de capacitación turística a través de la cantidad, su vinculación y contenido. De acuerdo al estudio de competitividad realizado recientemente en la localidad (UNSIJ, 2015), la evaluación de los planes fue negativa, con base a esto, se calificó también como negativa a ésta variable.

- Cuenta con un sistema de información que evalúe la satisfacción de los visitantes: Mide la existencia de algún instrumento de recolección de datos que evalúe el grado de satisfacción de los turistas. En visita en campo, se constató que sí se cuenta con un sistema de información de la satisfacción de los turistas (encuestas) que se aplica cotidianamente, por lo que esta variable se calificó como positiva.

- Indicadores de calidad de las evaluaciones de la satisfacción de los visitantes: Grado de calidad y fiabilidad en los indicadores de evaluación para arrojar el grado de satisfacción de los turistas. Se encontró que los indicadores de evaluación eran los adecuados, ya que además, al estar apegados al programa de "Pueblos Mágicos" existían guías para la elaboración de estos, mismas que se siguieron, por esta razón esta variable se califica como positiva.

De acuerdo a la ponderación de estas tres variables, el valor del componente de seguimiento de la calidad es igual a $66.7 \%$.

\subsection{Componente de percepción de la calidad y satisfacción del turista. Este componente se desintegró para su análisis en las siguientes variables:}

- Percepción del turista sobre los atractivos turísticos: Sobre los atractivos y los servicios que ofrece Capulálpam de Méndez, el $96.5 \%$ de los turistas encuestados los calificaron como muy buenos, mientras que el $3.4 \%$ los calificaron como malos. El $94.1 \%$ considera que el estado de conservación de los atractivos del destino es muy bueno, mientras que solo el $5.8 \%$ lo calificaron como en mal estado. La opinión en función del tipo de atractivo también fue muy buena: sobre los atractivos culturales el $96.0 \%$ de los turistas indicó que se encuentran en buen estado, la percepción sobre los atractivos naturales fue muy similar, ya que el $97.0 \%$ indicaron que se encuentran en muy buen estado. La variedad de los atractivos fue calificada de manera favorable por parte de los turistas, puesel $96.0 \%$ indicó que existe una gran variedad de atractivos (UNSIJ, 2015). Debido a lo anterior, esta variable se calificó como positiva.

- Percepción del turista sobre los establecimientos de hospedaje: Según datos de la UNSIJ (2015), existe una mayor preferencia del turista por el hospedaje en cabañas (52.0 \%) que en hotel (21\%), un $11.0 \%$ lo hace en tiendas de campaña, mientras que el $16.0 \%$ no se hospedo en el destino turístico. La percepción del turista acerca del hospedaje es favorable, pues el $98.2 \%$ de los turistas entrevistados indicaron que su estancia y la calidad del hospedaje fue muy buena. Debido a lo anterior, esta variable se calificó como positiva.

- Percepción del turista sobre el servicio de alimentación: El $43.4 \%$ de los turistas encuestados indicaron que consumieron sus alimentos y bebidas en un restaurante, el $37.3 \%$ indico que en el mercado, las fondas y cafeterías representaron un menor porcentaje. Respecto a su experiencia en estos establecimientos, el $97.3 \%$ de los turistas encuestados indicaron que la experiencia en el consumo de alimentos y bebidas, la calidad y la higiene de los mismos fue muy buena (UNSIJ, 2015). Debido a lo anterior, esta variable se calificó como positiva.

- Percepción del turista sobre los precios de los bienes y servicios: De acuerdo a la encuesta realizada por la UNSIJ (2015), el 92.9 \% de las personas encuestadas señalaron que la relación de la calidad con el precio del destino turístico fue aceptable. Por otra parte, el $93.1 \%$ indicaron que los costos de transportación fueron adecuados. De las personas encuestadas que utilizaron hospedaje en el destino, el $91.0 \%$ señalo que el precio por el servicio es aceptable. El $95.7 \%$ de 
los turistas que consumieron alimentos y bebidas en el destino, indicó que el precio pagado por el servicio fue aceptable. Debido a lo anterior, esta variable se calificó como positiva.

- Experiencia del turista: En relación a la experiencia del viaje, se encontró que la totalidad de personas encuestadas, indicó que la experiencia del viaje fue muy buena, el $96.4 \%$ señaló que la hospitalidad de los residentes fue muy buena, el $97.6 \%$ señalo que la experiencia de visitar los sitios naturales fue agradable. En relación a la variedad de actividades recreativas, el $97.6 \%$ indicó que la emoción de visitar este sitio fue muy alta y el $98.8 \%$ expreso que fue una experiencia diferente. El 89.0 \% de los turistas encuestados indicó que la disponibilidad de información turística durante el viaje fue muy buena (UNSIJ, 2015). Esta variable se evaluó como positiva, debido a que un alto porcentaje de turistas expresó que la experiencia en el destino fue muy agradable.

- Satisfacción y recomendación: Se encontró que el $98.8 \%$ de los turistas entrevistados indicaron que la satisfacción de visitar el destino fue muy alta y cubrió sus expectativas. Al compararlo con el mejor destino que hayan visitado, el $73.1 \%$ de los turistas consideraron que en cuanto a satisfacción el destino está muy cercano al mejor destino que han visitado, mientras que un 26.8 $\%$ consideraron que está muy lejano. El $93.9 \%$ de las personas indicaron que definitivamente volverían al destino turístico y el 96.4 \% señaló que recomendaría el destino a sus amigos (UNSIJ, 2015). Debido a lo anterior, esta variable se calificó como positiva.

De acuerdo a la ponderación de estas seis variables, el valor del componente de seguimiento de percepción de la calidad y satisfacción del turista es igual al $100 \%$.

El combinar los cuatro subíndices anteriores e integrándolos en uno mismo, ponderando un valor equitativo para todos, se obtiene una evaluación final para este componente del $63.3 \%$. Siendo que el $100 \%$ representa la calificación máxima alcanzable, el componente en términos generales recibe una evaluación ligeramente positiva.

\section{Conclusiones.}

La percepción del turista hacia el destino es bastante positiva, ya que considera que el destino es recomendable, barato y de calidad, al grado de recomendarlo o de volver a visitarlo, sin embargo, aunque la percepción del turista es buena, hace falta documentarla con la obtención de los distintivos y certificaciones legales que amparen esa calidad que ya la calidad la percibe el turista solo a posteriori. Contar con estas certificaciones de calidad, le darán al turista a priori, una idea de la calidad del servicio que va a recibir durante su estancia, incidiendo en su decisión de compra.

Las certificaciones también son importantes ya que permiten entre otros beneficios: estandarizar productos y servicios, entrar en un ciclo de mejora continua de la calidad de su producto, incrementar la reputación del destino, tener mayores posibilidades de compra y elevar notoriamente su competitividad respecto a otros destinos turísticos similares o incluso diferentes, como los de playa.

Respecto al capital humano, se encontró que el destino tiene capacitada a la mayoría del personal que atiende al turista, pero esta capacitación es solo a nivel técnico, no profesional universitario, esto supone una debilidad sobre todo a la hora de articular planes estratégicos a largo plazo. La carencia de esta fortaleza afectó no solamente al componente de calidad del capital humano, sino también repercutió en el componente de seguimiento de la calidad (específicamente en los planes de capacitación turística) y en el de la calidad de la oferta (falta de certificaciones). Por lo que esta debe ser otra carencia que deberá ser atendida a la brevedad.

Finalmente, mencionar, que en la presente evaluación, la gran mayoría de las variables fueron analizadas en función del criterio "cumple/no cumple" o sí, "existe/no existe", un análisis más profundo debería de indagar también sobre el grado de existencia o el cumplimiento. Desafortunadamente, la falta de recursos nos impidió realizar ambos tipos de evaluación mencionados en este apartado, pero nos pareció muy importante mencionarlo como propuesta para futuras investigaciones.

\section{Notas:}

1. "Un Pueblo Mágico es una localidad que tiene atributos simbólicos, leyendas, historia, hechos trascendentes, cotidianidad, en fin magia que te emanan en cada una de sus manifestaciones socio-culturales, y que significan hoy día una gran oportunidad para el aprovechamiento turístico" (SECTUR, 2015). 
2. El índice de satisfacción se elabora con las respuestas a cada uno de los reactivos de una encuesta de satisfacción aplicada a turistas. Las respuestas podían ser: excelente, bueno, malo y pésimo. La fórmula del Índice de Satisfacción (IS), considera una escala de 0 a 10 a partir de las 4 posibles respuestas (excelente, bueno, malo y pésimo) y se expresa como: IS $=[5+\{0.05 *(\% \text { Excelente }-\% \text { Muy Malo })\}+\{0.025 *(\% \text { Bueno }-\% \text { Malo })\}]^{*} 10$ (Cruz, Juárez, Cruz y Urciaga, 2015)

3. Cálculos propios realizados con base en los datos obtenidos del Plan Municipal de Desarrollo de Capulálpam de Méndez (2009) y al Diagnóstico Municipal de Salud (SSO, 2014:14).

\section{Bibliografía}

Chiavenato, I.

2000 Administración de Recursos humanos. Bogotá: Mc. Graw Hill.

Cruz, P., Juárez, J., Cruz, G. y Urciaga J.

2015 "Evaluación del visitante en el Pueblo Mágico de Loreto". Global Conference on Business and

Finance Proceedings, 10 (1): 1264-1270.

Dupeyras, A. \& MacCallum, N.

2013 "Indicators for Measuring Competitiveness in Tourism: A Guidance Document". Disponible en: http://dx.doi.org/10.1787/5k47t9q2t923-en: OECD Tourism Papers, 2013/02. OCDE PUblishing.

Fecha de acceso: 20 de junio de 2015.

García, I.

2013 Gestión de Recursos Humanos en las empresas turísticas (p. 324). Editorial Paraninfo.

García, I., Fernández, M. y Moreno, M.

2013 "El perfil ocupacional de los titulados en turismo dentro del sector turístico". Gran Tour: Revista

de Investigaciones Turísticas, 7 (Enero-Junio): 99-100.

González, A.

2000 "Evaluación de desempeño, la controversia sin fin". Actualidad. Psicológica, 274: 23-25.

Hellrigel, D., Jackson, S. \& Slocum, J.

2005 Administración, un enfoque basado en competencias (p. 384). Thomson, $10^{a}$ edición.

Koontz, H., Weihrich, H. \& Cannice, M.

2012 Administración: una perspectiva global. McGraw-Hill.

Lazarsfeld, P.

1958 Evidence and Inference in Social Research. Daedalus, 87 (4).

Municipio de Capulálpam de Méndez.

2009 "Plan Municipal de Desarrollo".

Pedro, A.

2015 "El reto de la competitividad y sostenibilidad para los destinos turísticos". Espacio I\&D., IV (7),

Febrero.

Secretaria de Turismo y Desarrollo Económico.

2013 "Indicadores básicos preliminares turistas en la Sierra Norte, Valles Centrales, Mixteca, Costa y

Papaloapam, que reportan los comités de ecoturismo y sociedades cooperativas".

Secretaria de Turismo.

2014 "Acuerdo por el que se establecen los Lineamientos generales para la incorporación y permanencia

al Programa Pueblos Mágicos". Diario Oficial de la Federación, Primera Sección.

Secretaria de Turismo.

2015 http://www.sectur.gob.mx/pueblos-magicos/ Fecha de acceso: 22 de junio de 2015.

Servicios de Salud de Oaxaca.

2014 "Diagnóstico Municipal de Salud” (p. 14).

Universidad Anáhuac México Norte.

2012 "Evaluación de desempeño de los destinos turísticos en el marco de los Convenios de Coordinación

en materia de Reasignación de Recursos".

Universidad de la Sierra Juárez.

2015 "Diagnóstico de Competitividad Turística del Destino Capulálpam de Méndez, Oax., Pueblo Mágico".

Recibido

$27 / 07 / 2015$

Reenviado

$24 / 01 / 2017$

Aceptado

$14 / 02 / 2017$

Sometido a evaluación por pares anónimos 